\title{
Infantile spasm: an overview
}

\author{
W. Donald Shields \\ UCLA School of Medicine, Division of Pediatric Neurology, \\ Los Angeles, CA 90095-1752, U.S.A.
}

Infantile spasm (IS) is the archetypal catastrophic childhood epilepsy syndrome. It is catastrophic for two reasons: first, it is difficult to control and second, it is strongly associated with mental retardation. In some cases, profound mental retardation is an inevitable consequence of the underlying disease; for example, lissencephaly. In other cases, the developmental outcome may be improved by controlling the seizures. Thus, early diagnosis and treatment is important. IS has a characteristic clinical presentation with flexor spasms beginning in the first year of life. The diagnosis of IS is confirmed by the electroencephalogram (EEG), which typically demonstrated hypsarrhythmia, modified hypsarrhythmia or multifocal spikewave discharges. A normal EEG should raise the possibility of one of the benign infantile seizure disorders such as benign neonatal convulsions, benign infantile familial convulsions or benign infantile myoclonus.

Having identified IS clinically and confirmed the diagnosis with EEG, each case must be carefully evaluated to determine the underlying etiology. There is a very extensive catalog of disorders that have been associated with IS (Table 1); many require sophisticated laboratory testing. However, about $70 \%$ of all patients will have a confirmed etiologic diagnosis following a careful history, physical and neurologic examination and magnetic resonance imaging scan. Of the remaining 30\%, less than half will have the diagnosis established by metabolic screening, genetic testing or lumbar puncture. As a general rule, these tests should be reserved for the $30 \%$ of undiagnosed patients. It

Correspondence: Prof. Dr. W. Donald Shields,

UCLA School of Medicine,

Division of Pediatric Neurology,

Los Angeles,

CA 90095-1752, U.S.A.

Fax: 3108255834 .

E-mail:wshields@mednet.ucla.edu

Received: December 10, 2003.

Accepted: December 12, 2003. is not necessary to perform these tests in all the patients.

The treatment of young children with IS is particularly vexing. It is a difficult disorder to treat but there is some evidence that early and effective treatment may alter the long-term developmental outcome. Of course, efficacy must be balanced against the risks of the therapy. Most of our standard anticonvulsant medications are not very effective for the treatment of IS and there are only two drugs with class 1 evidence of efficacy, adrenocorticotropic hormone (ACTH) and vigabatrin. IS is the only seizure syndrome where ACTH is a drug of choice, an intimation that IS is fundamentally different from other seizure disorders. However, ACTH has an unfavorable side effect profile. The majority of children treated with ACTH develops cushingoid obesity and become very irritable. However, all the children are at risk to develop one or more of the other important side effects including arterial hypertension, electrolyte imbalance, gastric ulcer, growth retardation, cardiomyopathy and immunosuppression with increased risk of infection. Vigabatrin is effective in the treatment of IS but also has an unfavorable side-effect profile because visual field constriction has been associated with its use. Clearly, an effective therapy with fewer serious side effects would be useful. Pyridoxine is a drug with a much more favorable side effect profile. Indeed, because of this, it has emerged as the drug of first choice for most patients with IS in Japan and in a few other centers around the world. However, it is not generally considered as a first-line medication by most epileptologists.

While Down syndrome is a fairly common cause of IS, it is also one of the few etiologies where is reasonably easy to achieve control (1). The spasms respond well to vigabatrin (2) or to ACTH (3). However, the paper by Carballo et al. (4) suggests that it may not be necessary to assume the risks of ACTH or vigabatrin in Down syndrome patients with IS and that pyridoxine may be particularly efficacious. Given the nature of the side effects associated ACTH and vigabatrin, it is reasonable to consider a trial of pyridoxine in patients with IS associated with Down syndrome. Indeed, it may be 
Table 1. Etiologies of infantile spasms

\begin{tabular}{lll}
\hline \hline Pyridoxine dependent seizures & Cardiac arrest & Pachygyria \\
Phenylkenonuria & Meningitis & Stroke \\
Maple syrup urine disease & Cerebral abscess & Leigh disease \\
Tumor & Transplacental infections & Hydranencephaly \\
Arteriovenous malformation & Trauma & Corpus callosum agenesis/dysgenesis \\
Sturge-Weber syndrome & Post cardiac surgery & Septo-optic dysplasia \\
Tuberous sclerosis & Neurofibromatosis & Schizencephaly \\
Biotinidase deficiency & Sebaceous nevus syndrome & Holoprosencephaly \\
Menkes disease & Incontinentia pigmenti & Multiple pineal cysts \\
Hyperammonemia disorders & Epidermal nevus syndrome & Periventricular leukomalacia \\
Nonketotic hyperglycinemia & Hydrocephalus & Band heterotopia \\
Cortical dysplasia & Miller-Dieker syndrome & Porencephaly \\
Focal cortical dysplasia & Down syndrome & Maternal toxemia \\
Hemimegalencephaly & Tuberous sclerosis & Encephalitis \\
Perinatal HIE & Aicardi syndrome & Near drowning \\
Near miss SIDS & Lissencephaly & Krabbe disease \\
& & \\
\hline
\end{tabular}

HIE: Hypoxic-ischemic encephalopathy; SIDS: Sudden infant death syndrome.

wise to consider a trial of pyridoxine in IS patients with other etiologies before starting either ACTH or vigabatrin.

\section{References}

1. Stafstrom CE, Konkol RJ. Infantile spasms in children with Down syndrome. Dev Med Child Neurol 1994; 36: 576-585.

2. Nabbout R, Melki I, Gerbaka B, Dulac O,
Akatcherian C. Infantile spasms in Down syndrome: good response to a short course of vigabatrin. Epilepsia 2001; 42: 1580-1583.

3. Escofet C, Poo P, Valbuena O, Gassio R, Sanmarti FX, Campistol J. Infantile spasms in children with Down's syndrome. Rev Neurol 1995; 23: 315-317 (in Spanish).

4. Caraballo RH, Cersósimo RO, Garro F, Kesler K, Fejerman N. Infantile spasms in Down syndrome: a good response to vitamin B6. J Pediatr Neurol 2004; 2: 15-19. 